\title{
Deformation microstructure of TRIP/TWIP Steels at the early deformation stages
}

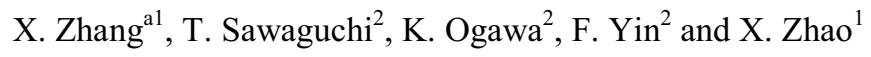 \\ ${ }^{1}$ Key Laboratory for Anisotropy and Texture of Materials, Northeastern University, Shenyang 110004, People's \\ Republic of China \\ ${ }^{2}$ Exploratory Materials Research Laboratory for Reliability and Safety, National Institute for Materials Science, \\ Tsukuba 305-0047, Japan
}

\begin{abstract}
Deformation microstructures of austenitic $\mathrm{Fe}-30 \mathrm{Mn}-(6-\mathrm{x}) \mathrm{Si}-\mathrm{xAl}(\mathrm{x}=2,3$ mass \%) steels have been investigated at different nominal strains by the combined use of atomic force microscopy (AFM), electron backscatter diffraction (EBSD) and transmission electron microscopy (TEM). Three kinds of deformation products, i.e. planar dislocation bands, $\varepsilon$ martensite plates and deformation twins are commonly formed on $\{111\}$ habit planes and exhibit a plate-like morphology in AFM images. At the very early stage of plastic deformation the main microstructure of Fe-30Mn-4Si-2Al steel is $\varepsilon$ martensite. Some of the $\varepsilon$ maretensite plates transform to the deformation twins with increasing tensile strain. On the other hand, the main microstructures of Fe-30Mn-3Si-3Al are planar and wavy dislocations at the onset of plastic deformation. The deformation twins generate and increase as deformation proceeds. In spite of the quite different microstructures between the two alloys, they exhibit the similar deformation behavior with strain hardening rates comparable to each other.
\end{abstract}

\section{Introduction}

Austenitic Fe-high Mn steels of low stacking fault energy have been paid much attention because of a good combination of high strength and ductility associated with TRIP (Transformation Induced Plasticity) and TWIP (Twinning Induced Plasticity) effects. During the past decade, various TRIP/TWIP steels, such as Fe-Mn-Si-Al, Fe-Mn-C and Fe-Mn-Al-C etc [1-3], with a high work hardening capacity and exceptional ductility have been developed [4-6]. For example, it was reported that an Fe-25Mn-3Si-3Al TWIP steel showed a relatively low flow stress of $\mathrm{R}_{\mathrm{P} 0.2}=280 \mathrm{MPa}$, a moderate tensile strength of $650 \mathrm{MPa}$, and the extremely high elongation to failure of $\varepsilon_{\mathrm{f}}=95 \%$ [3]. They are thus potentially attractive for automobile applications involving press-formed parts for energy absorption or for structural reinforcement [7].

Despite Extensive research of TWIP Steel since its discovery, the mechanism of the high strain hardening behavior of this austenitic steel remains uncertain. Most authors associated the strain hardening with deformation twinning, and interpreted that twin boundary increases the work hardening rate in these alloys by creating strong barriers to further plastic flow [8-11]. However, some investigations attributed the hardening to dynamic strain aging (DSA) mechanisms which is caused by deceleration of dislocations due to carbon- or nitrogen-vacancy pairs [12-14]. Recently, R.Ueji et al suggested that the reason for the large ductility lies not only on the twinning but also on the suppressed dynamic recovery due to low stacking fault energy [15]. F. Hamdi reported that slip barriers such as formation of Lomer-Cottrell (LC) locks can also produce a high strain hardening comparable to TWIP effect, and concluded that the slip planarity play an important role on the strain hardening [20].

A deeper understanding is needed for the further development on the TWIP steels. Recently much research has been focused on deformation microstructures responsible for mechanical properties [16-19]. D. Barbier et al described the deformation mechanisms by means of texture and microstructure evolutions, and stressed the dependence of twinning on grain size and grain orientation [16]. P Yang et al studied the effect of the crystallographic rotation due to slip and the Schmid factor on deformation twinning [17].

a e-mail: zhang.xin@nims.go.jp

This is an Open Access article distributed under the terms of the Creative Commons Attribution-Noncommercial License (http://creativecommons.org/licenses/by-nc/3.0/), which permits unrestricted use, distribution, and reproduction in any noncommercial medium, provided the original work is properly cited. 
Most of the works have been done at large deformation, in spite of the fact that the extraordinarily high work hardening appears at early deformation stages. Up to now only the limited numbers of reports have been made on the microstructures of TWIP Steels in the early plastic deformation stages [16]. In the literature, not only the deformation twins but also planar dislocations and forest dislocations have been observed at early deformation stages. In order to reveal the detailed deformation mechanisms, therefore, it is of crucial importance to analyze the dominant deformation modes and their spatial distributions at the early plastic deformation stages.

In order to clarify the deformation modes controlling deformation behavior at early stages, we made a comparative study between two austenitic Fe-Mn-Si-Al steels: i.e. Fe-30Mn-(6-x)Si-xAl ( $\mathrm{x}=2$ and 3 in mass \%). These alloys were selected for the following two reasons. First, these alloys contain no carbon, so that it is unnecessary to consider the DSA that is related to the motion of interstitial atoms. Secondly, in spite of the similar chemical compositions, their deformation microstructures previously reported make a marked contrast: Fe-30Mn-3Si-3Al alloy is dominated by TWIP effect and Fe-30Mn-4Si-2Al with the combined contributions of TRIP and TWIP effects. Comparison has been conducted between $\varepsilon$ martensitic transformation and deformation twinning in their effect on deformation characteristics. The deformation microstructures of the steels have been investigated by the combined use of atomic force microscopy (AFM), Transmission electron microscopy (TEM) and electron backscatter diffraction (EBSD).

\section{Experimental}

The alloys were prepared by induction furnace melting, and the chemical composition (mass percent, \%) was Fe$30 \mathrm{Mn}-(6-\mathrm{x}) \mathrm{Si}-\mathrm{xAl}(\mathrm{x}=2,3)$. In this paper, hereafter the alloys were referred to as $\mathrm{A} 12, \mathrm{Al} 3$ using mass $\%$ of $\mathrm{Al}$. The specimens were solution treated at $1000^{\circ} \mathrm{C}$ for $3 \mathrm{~h}$ followed by water quenching after hot rolling and forging at $1000^{\circ} \mathrm{C}$. The high manganese content stabilizes the austenitic phase at room temperature and decreases the SFE, mechanical twinning and martensite transformation occur during deformation at room temperature $[21,22]$.

Tensile tests were conducted at room temperature by applying a strain rate of $0.03 \mathrm{~mm} / \mathrm{min}$. The tensile samples were cut parallel to the roll direction (RD). The gauge length was $15 \mathrm{~mm}$ and the width was $10 \mathrm{~mm}$. Al2 specimen was elongated by $2.0 \%, 5.8 \%, 10.4 \%$, and $30 \%$. Al3 specimen was elongated by $1.7 \%, 4.3 \%$, and $10.3 \%$.

Microstructural observations were performed with atomic force microscopy (AFM), electron backscatter diffraction (EBSD) and transmission electron microscope (TEM). The AFM and EBSD samples were mechanically polished, then electropolished to obtain smooth surfaces and diminish the extra induction of the surface deformation microstructure during the preparation procedure. AFM measurements were performed to analyze quantitatively the surface relief and reveal the microstructural evolution during tensile deformation. The EBSD measurement by scanning electron microscopy (SEM) was conducted on a Carl-Zeiss LEO-1550 Schottky field-emission instrument, equipped with a Tex-SEM Laboratories orientation-imaging microscope (OIM) system.

\section{Result}

\subsection{Mechanical Properties}

Fig. 1 shows the stress-strain curves for the sample A12 and A13. It can be seen that A12 has higher flow stresses than $\mathrm{Al} 3$ at all the strains measured (up to $10 \%$ ). The $0.2 \%$ proof stresses are $245 \mathrm{MPa}$ for $\mathrm{Al} 2$ and $220 \mathrm{MPa}$ for $\mathrm{Al} 3$, respectively. The deformation behaviors of the Al2 and Al3 specimens are comparable to previous reports [3], in which the combined TRIP/TWIP effect and TWIP effect, respectively, are considered to be responsible for the deformation behaviors. It should be noted here that they exhibit the similar strain hardening behavior, although A12 shows a slightly higher strain hardening rate comparable to Al3.

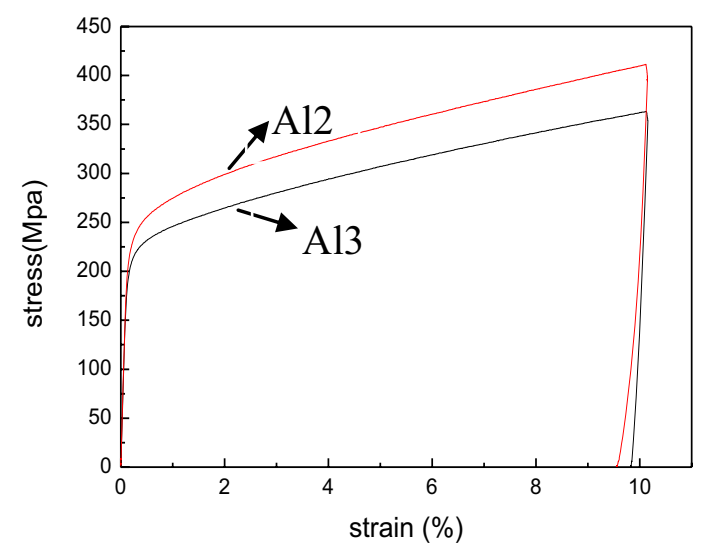

Fig. 1 Stress-strain curves of Al2 and Al3 alloys 


\subsection{Microstructure}

\subsubsection{Stepwise AFM observations}

Fig. 2 and Fig. 3 show the AFM images of Al2 and Al3 alloys obtained by different elongation and corresponding cross-section profile along the white line in the AFM images, respectively. Arrows indicate a particle found on the specimen surface, which was used to determine an identical point for the observations. Parallel bands were formed both in Al2 and Al3, which are caused either by the planar dislocation band, the stress induced $\varepsilon$ martensite or deformation $\gamma$ twin. It was clearly seen that the number of parallel bands increased with the increasing elongation. Surface relief angle of bands indicated by labels 1-5 were measured for each specimen, as shown in Table 1 and 2.
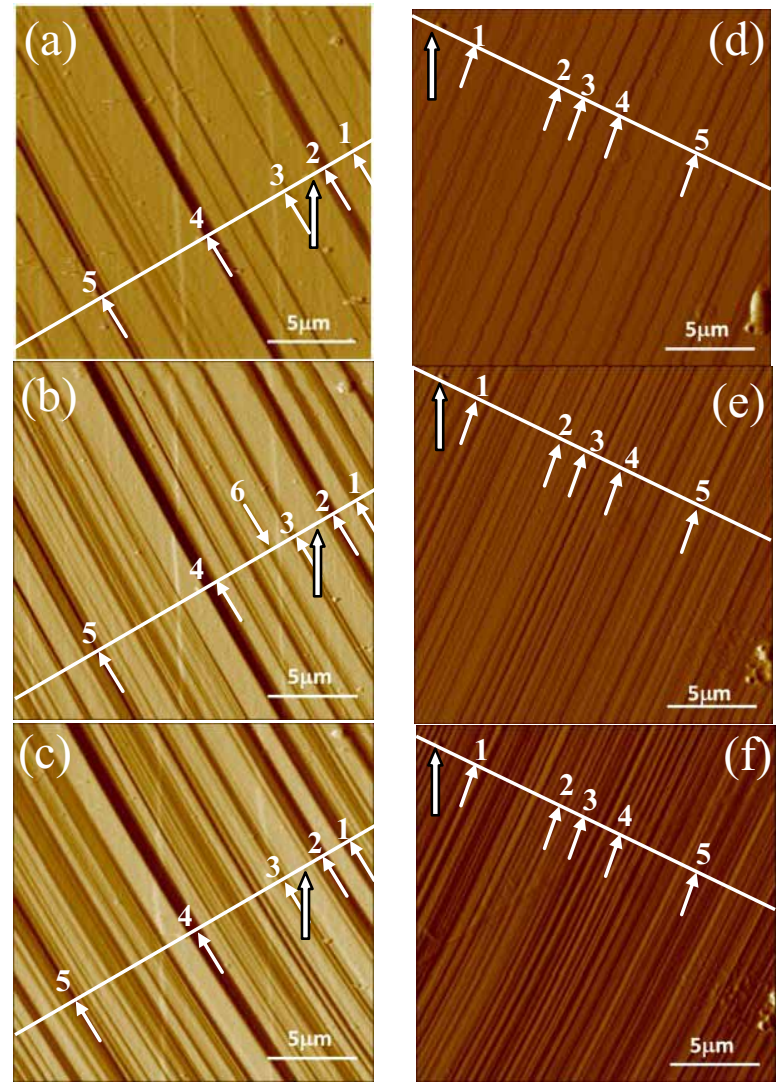

Fig. 2 AFM micrographs of Al2 and Al3 alloys under different elongation: (a) Al2 elongated by 2.0\%; (b) Al2 elongated by $5.8 \%$; (c) Al2 elongated by $10.4 \%$; (d) Al3 elongated by $1.7 \%$; (e) Al3 elongated by $4.3 \%$; (f) Al3 elongated by $10.3 \%$
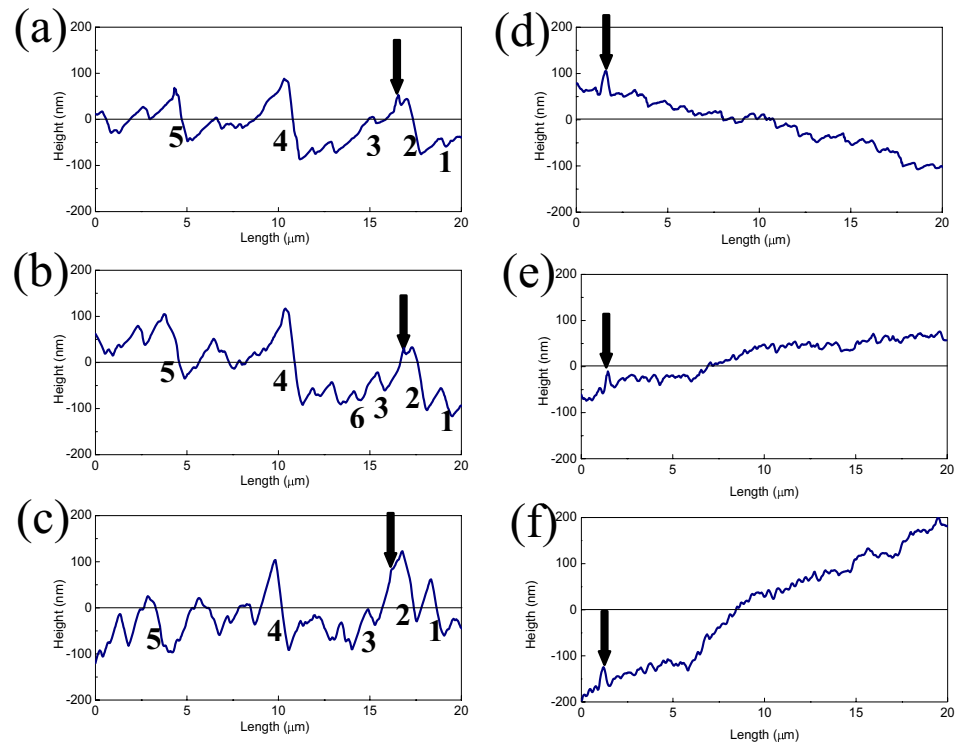

Fig. 3 Surface relief corresponding to the AFM micrographs from Fig.2: (a) Al2 elongated by 2.0\%; (b) Al2 elongated by $5.8 \%$; (c) Al2 elongated by $10.4 \%$; (d) Al3 elongated by $1.7 \%$; (e) Al3 elongated by $4.3 \%$; (f) Al3 elongated by $10.3 \%$ 
The orientations of the grain observed were measured by EBSD on the specimens prior to elongation: $\mathrm{RD} / /\left[\begin{array}{lll}12 & -23 & -3\end{array}\right], \mathrm{ND} / /\left[\begin{array}{lll}-5 & -6 & 26\end{array}\right]$ for $\mathrm{Al} 2$ and $\mathrm{RD} / /\left[\begin{array}{lll}11 & -3 & 9\end{array}\right], \mathrm{ND} / /\left[\begin{array}{lll}-12 & -5 & 13\end{array}\right]$ for $\mathrm{Al}$, respectively. The shear systems responsible for the banded structures were identified as $\left(\begin{array}{lll}-1 & 1 & -1\end{array}\right)\left[\begin{array}{lll}1 & -1 & -2\end{array}\right]$ for Al2 and (-1 $\left.11-1\right)\left[\begin{array}{llll}1 & 2 & 1\end{array}\right]$ for $\mathrm{Al} 3$, respectively, from the grain orientations, the direction of $\{111\}$ traces and their surface slopes. The theoretical values for martensite and deformation twin were then calculated from the geometric relationship [23]. Those for Al2 are $10.8^{\circ}$ and $18.7^{\circ}$, respectively. It was found by comparing these values with experimental values in Table 1 that the surface tilt angles at $2.0 \%$ are close to the theoretical value the $\varepsilon$, and increase with increasing the tensile strain to reach the theoretical value of the deformation twin. At the same time the new bands with the surface tilt angles close to the theoretical value of the $\varepsilon$ martensite, for example, the band indicated by label $6\left(10.1^{\circ}\right)$, are successively nucleate in the austenite matrix, as seen in Figs. 2 (a) to (c). These results indicate that the dominant deformation mode of the Al2 alloy is the stress-induced martensitic transformation for the smaller strain and alters into the deformation twinning as the deformation proceeds.

Table 1 Surface tilt angles of banded structures in Al2 under different elongation $(2.0 \%, 5.8 \%, 10.4 \%)$

\begin{tabular}{cccc}
\hline Number & Angle $(2.0 \%)$ & Angle $(5.8 \%)$ & Angle (10.4\%) \\
\hline 1 & $8.6^{\circ}$ & $12.0^{\circ}$ & $20.9^{\circ}$ \\
2 & $12.6^{\circ}$ & $19.9^{\circ}$ & $24.0^{\circ}$ \\
3 & $11.3^{\circ}$ & $14.2^{\circ}$ & $21.5^{\circ}$ \\
4 & $21.5^{\circ}$ & $24.4^{\circ}$ & $24.5^{\circ}$ \\
5 & $15.8^{\circ}$ & $17.1^{\circ}$ & $25.5^{\circ}$ \\
\hline
\end{tabular}

The theoretical values for martensite and deformation twin are $3.9^{\circ}$ and $7.7^{\circ}$, respectively, for the grain observed in Figs. 2(d) to (f). Most of the bands were too thin to be uniquely identified by referring to the theoretical value of the surface tile angle. However, some plates with relatively large thicknesses could be selected for the quantitative surface analysis. The values of their surface tilt angles at various strains are listed in Table 2. All of the selected plates have the surface tilt angles comparable to the theoretical values of the deformation twins.

Table 2 Surface tilt angles of banded structure in Al3 steel under different elongation $(1.7 \% ; 4.3 \% ; 10.3 \%)$

\begin{tabular}{cccc}
\hline Number & Angle $(1.7 \%)$ & Angle $(4.3 \%)$ & Angle $(10.3 \%)$ \\
\hline 1 & $5.3^{\circ}$ & $8.1^{\circ}$ & $7.5^{\circ}$ \\
2 & $6.4^{\circ}$ & $7.4^{\circ}$ & $6.1^{\circ}$ \\
3 & $6.3^{\circ}$ & $8.9^{\circ}$ & $6.1^{\circ}$ \\
4 & $9.8^{\circ}$ & $8.9^{\circ}$ & $5.6^{\circ}$ \\
5 & $8.7^{\circ}$ & $8.1^{\circ}$ & $7.4^{\circ}$ \\
\hline
\end{tabular}

\subsubsection{EBSD Observations}

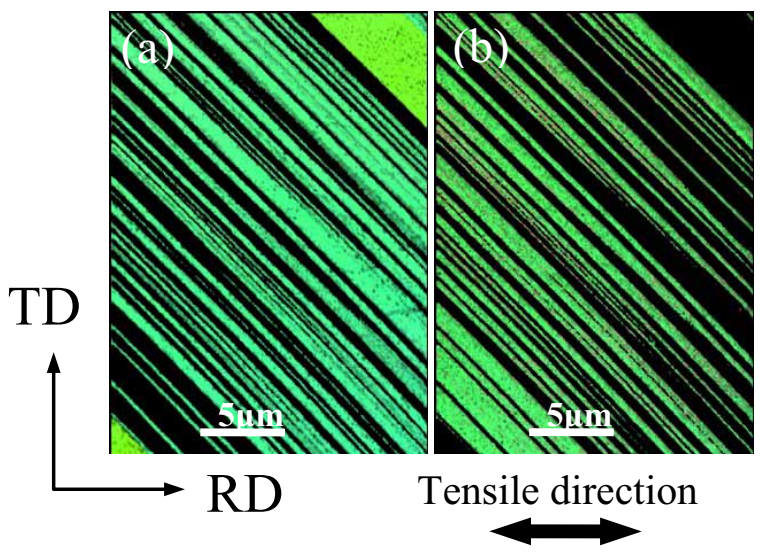

Fig. 4 EBSD map of Al2 alloy elongated by $10 \%$

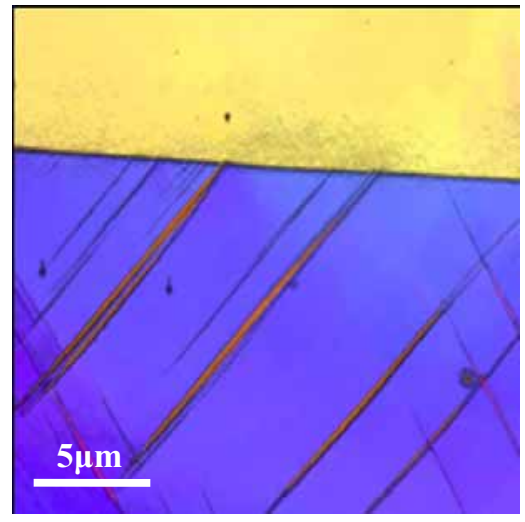

Fig. 5 EBSD map of Al3 alloy elongated by $10 \%$

The existence of $\gamma$ phase and $\varepsilon$ phase in A12 and Al3 alloys were also observed by EBSD. Fig. 4 shows deformation microstructure of A12 elongated by $10 \%$. The formation of tensile induced $\varepsilon$ martensite was clearly detected. The surface area fraction of $\gamma$ phase and $\varepsilon$ phase was $56.4 \%$ and $43.6 \%$, respectively. Different from the AFM results at the same elongation (10\%), no deformation twin was observed and the $\varepsilon$ martensite was still the dominant deformation mode. Fig. 5 shows the deformation microstructure observed by EBSD on the Al3 specimen tensile deformed by $10 \%$. As a result of crystallographic analysis, it was found that the bands were formed on two $\{111\}$ habit planes. They were identified as deformation twins of two different habit plane 
variants. It was found that the area fraction of deformation twins was 1.6\%. As seen in Fig. 2(f), AFM image showed much more banded surface markings at the same strain. Further AFM observations at various grains and low magnitude optical microscopic observations revealed that the banded structures are formed in most of grains and spread all over each grain. By comparing the AFM images, for example Fig. 2(f), and the EBSD image in Fig. 5, it is clear that the bands observed in AFM images can not be interpreted only by the deformation twins.

As mentioned in the above, EBSD images at $10 \%$ demonstrated the same deformation modes observed in AFM: stress-induced martensitic transformation in A12, and $\gamma$ deformation twinning in Al3. However, the deformation twins were not observed in A12 different from AFM results, and the area fraction of the deformation twins in Al3 is much less than those observed in AFM. The deformation twins in Al2 were observed when the alloy was further elongated to $30 \%$, as indicted by arrow in Fig. 6 . The deformation twins are formed inside the $\varepsilon$ martensite plates. This results and the AFM results indicate that the $\varepsilon$ martensite transforms into the deformation twin by further deformation.
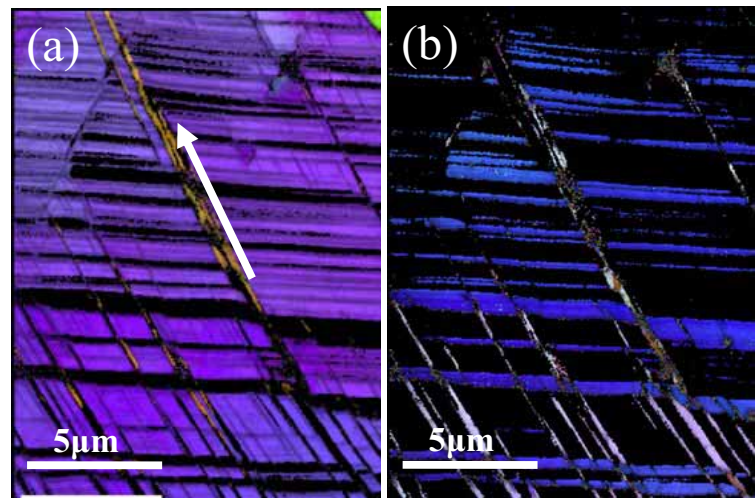

Fig. 6 EBSD map of A12 alloy elongated by $30 \%$ : (a) $\gamma$ phase (b) $\varepsilon$ phase

\subsubsection{TEM Microstructures}

In order to directly identify the deformation modes in two specimens, TEM observation were made at very early stages $(0.2$ to $0.3 \%)$ and at about $10 \%$. Fig. 7 shows typical microstructure of Al2 specimen tensile deformed to $10 \%$ and the corresponding electron diffraction pattern taken from the encircled area. The plates running obliquely in Fig. 7 were $\varepsilon$ martensite, as confirmed by diffraction pattern. Thin plates belonging to the second (11-1) habit plane variant are also formed. They collapse to the thicker primary variant on the (1-11) habit plane, and their growth is interrupted. The stacking faults on a third $\{111\}$ plane can be seen in the areas indicated by arrows. The stacking faults are enclosed within areas delineated by the primary and secondary $\varepsilon$ martensite plates. A number of the TEM observations were carried out at various locations of the same specimen, and the $\varepsilon$ martensite plates were frequently observed, but the deformation twin plates were scarcely found. From these results, it can be concluded that the dominant deformation mode in the Al2 alloy is the stress-induced $\gamma \rightarrow \varepsilon$ martensitic transformation.



Fig. 7 TEM bright-field micrograph and corresponding electron diffraction pattern of Al2 alloy elongated by $10 \%$.

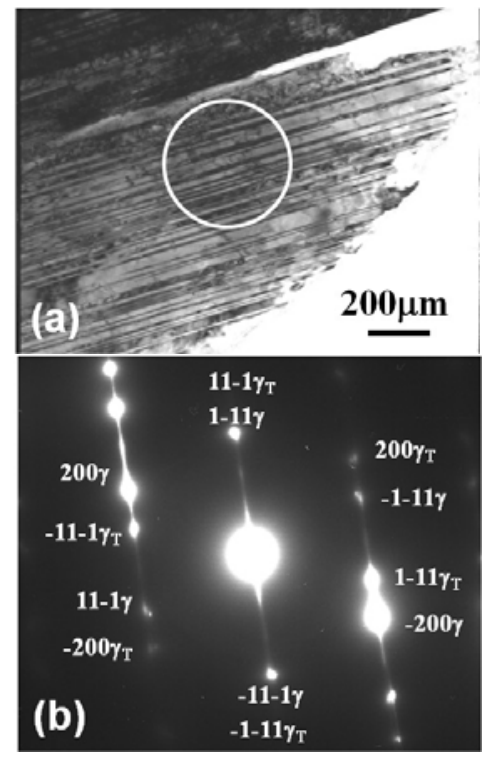

Fig. 8 TEM bright-field micrograph (a) and corresponding electron diffraction pattern (b) of Al3 alloy elongated by $10 \%$ 
Fig. 8 shows the existence of the deformation twin plates in Al3 alloy tensile deformed to 10\%. From Fig. 8, the specimen shows a lamella structure consisted of the very thin deformation twin plates with the thicknesses of about $10 \mathrm{~nm}$ and the remaining the $\gamma$ austenite matrix in between them.

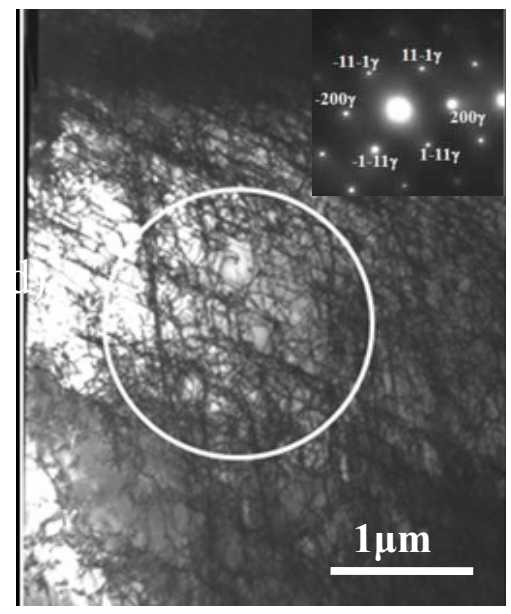

Fig. 9 TEM bright-field micrograph of $\mathrm{Al} 3$ alloy elongated by $10 \%$

Another TEM picture taken inside a different grain of the same specimen showed a quite different structure, as illustrated in Fig. 9. No deformation twin was observed, and a considerable amount of dislocations appear, instead. Among them, two dislocation groups can be seen: dislocations lying on (-1-11) planes and those on $(-100)$ planes. The former dislocations are observed as the thick strait lines with a dark contrast. Because the $(-1-11)$ plane is observed in the edge-on direction, the straight lines are considered as planar dislocation bands. The possible explanation for the latter group of dislocations on (-100) planes are the Lomer-Cottrel lock along [01-1] direction. There are also a number of wavy dislocations between these two dislocation groups.

\section{Discussion}

There are advantages and disadvantages in each of AFM, EBSD, and TEM, when analyzing the plate-like deformation products in TRIP/TWIP steels. TEM is a direct phase identification method and has the highest spatial resolution among above three techniques, but its observing area is limited. AFM and EBSD enable us to investigate the spatial distribution of the plates. The spatial resolution of AFM is higher than EBSD, but the phase identification using the AFM surface analysis is a kind of indirect method. The EBSD is a direct phase identification method, but, its spatial resolution is limited by its spot size.

In this section, we determine the dominant plastic deformation mode by comparing the results from AFM, EBSD and TEM. For A12 specimen, there is a significant difference between AFM and EBSD. Some of the banded surface markings were identified as deformation twins in Al2 alloy at 5.8\%, as shown in Fig. 2(b). However, the strain where the deformation twins were observed in EBSD (elongated by 30\%, shown in Fig6(a)) is much larger than that in AFM. There are following three possible reasons for this.

1. The deformation process is affected by the grain orientation. The higher the Schmid factor for the primary $<112>$ shear is, the faster the change in the deformation mode from the $\varepsilon$ martensite to the deformation twin occurs.

2. Because of lower spatial resolution of the EBSD than AFM, the plates with very small thickness cannot be detected. The EBSD spot size used in this study is $50 \mathrm{~nm}$. Therefore, the plates with thicknesses smaller than this level are not be shown in the EBSD images. On the other hand, in AFM images, for example, in Fig. 2(f), the very thin plates are also observed.

3. The surface effect is also one of important reasons. The specimen surface can be a favorable site for the growth of the plate-like deformation products. In bulk condition, however, the growth of the plates is more restricted because the change in the outer shape of the grain is constrained by surrounding grains. In case of AFM observation, there was no additional surface polishing after tensile deformation. As a consequence, there is a possibility that the microstructure contains additional plates caused by the surface effect. On the other hand, the EBSD specimens were subjected to mechanical and subsequent electrolytic polishing after tensile deformation.

The Schmidt factor for the martensite / deformation twin plates observed by AFM in Fig. 2 is 0.453 , while the Schmidt factors for the plates observed by EBSD in Fig. 5 are 0.494 and 0.411 for the primary and secondary habit plane variants, respectively. They are not very different to each other. Therefore, the first item (Schmidt 
factor) is ruled out from the possible reasons for the different deformation mode between AFM and EBSD. TEM observations have showed no clear evidence for existence of deformation twins in $\mathrm{Al} 2$ at $10 \%$ so far.

Combining AFM, EBSD, and TEM results, it may conclude that surface effect is the most plausible reason for the difference between AFM and EBSD, and the main microstructure of Al2 alloy is $\varepsilon$ martensite at $10 \%$. The $\varepsilon$ maretensite plate was also found in the Al2 specimen at quite a small strain of $0.29 \%$, indicating that the martensitic transformation is dominant at the very early stage of plastic deformation. It should also be noted that the deformation mode shifts from stress-induced transformation to deformation twinning as the deformation proceeds, although the shifting point is different between AFM results and EBSD results due to the surface effect in AFM observations.

Another distinction between AFM and EBSD are seen in the amount of plates in A13 at $10 \%$. While AFM studies showed a large number of deformation plates in $\mathrm{Al} 3$ alloy elongated by $10 \%$ (Fig. 2(f)), EBSD result of the sample at same elongation contained only a few deformation twins (Fig5). One of the reasons for this difference is the surface effect, too. In addition to this, TEM results showed that both deformation twin and planar dislocation were formed in Al3 alloy elongated by $10 \%$, implying that some deformation bands observed in $\mathrm{Al} 3$ alloy under AFM may be planar dislocation bands. TEM observations were also carried out on $\mathrm{Al} 3$ alloy strained by $0.19 \%$ and $3 \%$, though they are not shown in the present article. No deformation twin was found at $0.19 \%$, but stacking faults and dislocation arrays appeared, instead. Thin deformation twins were observed at 3\%, but dislocations were still dominant at this strain level. Some of the dislocations were extended and built planar dislocation bands on $\{111\}$ planes and the others show wavy configurations. From these results, the dominant deformation mode in Al3 is considered to be the dislocation glide at small strains less than $10 \%$. Deformation twinning generates during further elongation and increases with increasing the strain to become the dominant deformation mode above $10 \%$.

It is notable that both $\mathrm{A} 12$ and $\mathrm{A} 13$ alloys with quite different microstructures mentioned above show similar trends in work hardening at early deformation stage. The dominant deformation mode is the stressinduced $\gamma \rightarrow \varepsilon$ martensitic transformation for Al2 steel and the main deformation sources for Al3 steel is the planar or wavy dislocations. Although contribution of the deformation twinning increases with increasing the strain in the latter alloy, the high work hardening at the early deformation stages cannot simply be interpreted by the deformation twinning.

F. Hamdi and S. Asgari have performed a detailed study on the evolution of microstructure during simple compression testing of two fcc polycrystals and showed that deformation twinning may not be the sole cause of linear hardening in low stacking fault energy fcc polycrystals [20]. They suggested that a more comprehensive model should consider slip planarity, that may be caused by low SFE [24, 25], as the main prerequisite for the high work hardening behavior. The concept of planarity is also applicable to the deformation behavior of A12 and Al3 alloys. Three kinds of planar deformation products, i.e. $\varepsilon$ martensite plates, deformation twins and planar dislocation bands, are commonly related to the movement of Shockley partial dislocations and the subsequent intrusion of stacking faults. The former two plates can be described as the ordering of stacking faults: the ordering on every other $\{111\}$ plane results in the $\varepsilon$ martensite and that on every $\{111\}$ plane results in deformation twinning. On the other hand, the planar dislocation band are made by the movement of extended dislocations, which may be randomly but densely distributed on the neighboring $\{111\}$ planes.

The planarity may promote the work hardening by i) inhibition of cross-slip process, ii) intersection of the plates, iii) interaction of the plates with boundaries. The cross-slip occurs by the pinching of partial dislocation in their original slip plane and their subsequent extension on the cross-slip plane, during the deformation of fcc metals [26, 27]. A large separation between the partials (low SFE) inhibits cross-slip and causes dislocations to organize themselves into planar arrays or planar slip bands [28]. Sessile dislocations such as Lomer-Cottrel lock are also formed owing to the cross-slip of extended dislocations, resulting in a high work hardening. The various plates also act as the obstacles against the motion of partial or complete dislocations on the other slip planes. Therefore, the intersection of the plates is regarded as one of the important factors affecting on the work hardening. The penetration of dislocations from one grain to the neighboring grain is also an important key for work hardening, because the grain boundary is a strong barrier against the motion of dislocations. For all the above three plate-like deformation products, the interaction between plates and boundaries can be described as that between Shockley partials and boundaries. This may be the reason for the similarity in deformation behavior between $\mathrm{Al} 2$ and $\mathrm{Al}$, in spite of contrastive deformation microstructures.

\section{Conclusion}

The dominant deformation sources are the stress-induced $\gamma \rightarrow \varepsilon$ martensitic transformation for the TRIP/TWIP steel (Fe-30Mn-4Si-2Al) at the very early stage of plastic deformation (at the tensile strain of $0.29 \%$ ). Some of $\varepsilon$ martensite plates transform into the deformation twins by further deformation, but the $\varepsilon$ martensite is still the dominant deformation mode at $10 \%$. On the other hand, no significant deformation twin was detected in the TWIP (Fe-30Mn-3Si-3 Al) steel at the onset of plastic deformation (0.19\%). Instead of this, planar and wavy 
dislocations coexist in the alloy. Deformation twinning becomes one of the dominant deformation modes at $10 \%$, but the planar and wavy dislocations still coexist as the other deformation modes depending on the grain observed. In spite of the quite different microstructures between the two alloys, they exhibit the similar deformation behavior with strain hardening rates comparable to each other. The fact indicates that the planarity of the martensite, twins and slip bands should commonly promote the work hardening by inhibition of cross-slip process, intersection of the plates, and interaction of the plates with boundaries.

\section{Acknowledgements}

We would like to thank Materials Manufacturing and Engineering Station, NIMS for the materials processing, and Dr. Kikuchi, Mr. Koyama for their experimental assistance and fruiteful discussions. A part of the present work was funded by Japan Society for the Promotion of Science (JSPS), Grant-in-Aid for Scientific Research (B) and New Energy and Industrial Technology Development Organization (NEDO).

\section{References}

[1] Grassel O, Kruger L, Frommeyer G, Int J. Plasticity 16, 1391-1409(2000)

[2] Scott C, Allain S, FaralM, et al, LaRevue deMetallurgie-CIT 6, 293-302(2006)

[3] Frommeyer G, Udo Brux, Peter Neumann, ISIJ International 43, 438-446(2003)

[4] O. Grassel, G. Frommeyer, Materials Science and Technology 14, 1213-1217(1998)

[5] Georg Frommeyer, et al, ISIJ International 43, 438-446(2003)

[6] O.Grassel, et al, J. PHYS. IV FRANCE 7, 383-387(1997)

[7] O.Bouaziz, et al, Scripta Materialia 58, 484-487(2008)

[8] I. Karaman, A. Sehitoglu, A.J. Beaudoin, Y.I. Chumlyakov, H.J. Maier, C.N. Tomé, Acta Mater 41, 20312047 (2000)

[9] L. Rémy, Acta Metall 26, 443-451(1978)

[10] L. Rémy, Metall. Mater. Trans. A 12A, 387-408(1981)

[11] S. Allain, J.P. Chateau, O. Bouaziz, M. Legros, X. Garat, Characterization of the mechanical twinning microstructure in a high manganese content austenitic steel,, Proceeding of the International Conference on TRIP-Aided High Strength Ferrous Alloys, Ghent, Belgium, 2002., 75-78

[12] Y.N. Dastur, W.C. Leslie, Metall. Mater. Trans. A 12A, 749-759 (1981)

[13] B. Hutchinson, N. Ridley, Scripta Mater 55, 299-302 (2006)

[14] T.S. Shun, C.M.Wan, J.G. Byrne, Acta Metall. Mater 40, 3407-3412(1992)

[15] R. Ueji, et al, Scripta materalia 59, 963-966(2008)

[16] D. Barbier, et al, Materials Science and Engineering 500, 196-206(2009)

[17] P Yang, et al, Scripta Materialia 55, 629-631(2006)

[18] Bracke L, et al, ACTA MATERIALIA 57, 1512-1524(2009)

[19] Li Qian,et al, Shanghai Metal 30, 8-11(2008)

[20] F. Hamdi, S. Asgari, Metals\&Materials Society and ASM International 39, 294-303(2008)

[21] C. Scott, N. Guelton, S. Allain, M. Farral, P. Cugy, Proceeding of the MS\&T’05 Conference., Pittsburgh, PA, USA, 2005

[22] S. Allain, J.P. Chateau, O. Bouaziz, S. Migot, N. Guelton, Mater. Sci. Eng. A-Struct 387, 158-162(2004)

[23] N. Bergeon, S. Kajiwara, T. Kikuchi, Acta mater 48, 4053-4064(2000)

[24] V. Gerold and H.P. Karnthaler, Acta Metall, 37, 2177(1989)

[25] S.I. Hong and C. Laird, Acta Metall, 38, 1581(1990)

[26] J. Friedel: in Dislocations and Mechanical Properties of Crystals, J.C.Fisher, W.G. Johnston, R. Thomson, and T. Vreeland, Jr., eds., John Wiley and Sons, Inc., Chapman and Hall, Ltd., New York, 1957, pp.330-32.

[27] J.P. Hirth and J. Lothe: Theory of Dislocations, McGraw-Hill, New York, NY, 1968, pp. 733-36.

[28] Aashish. Rohatgi, et al, Metallurgical and Materials Transactions A 32, 135-145(2001) 